\title{
Fully interferometric three-dimensional imaging spectrometry using hyperbolic-type volume interferogram
}

\author{
Tetsuya Hashimoto, ${ }^{1, *}$ Akiko Hirai, ${ }^{2}$ and Kyu Yoshimori ${ }^{1}$ \\ ${ }^{1}$ Department of Electrical Engineering and Computer Science, Graduate School of Engineering, \\ Iwate University, Ueda 4-3-5, Morioka, Iwate 020-8551, Japan \\ ${ }^{2}$ National Institute of Advanced Industrial Science and Technology (AIST), National Metrology Institute of Japan, \\ Tsukuba Central 3, 1-1-1 Umezono, Tsukuba, Ibaraki 305-8563, Japan \\ ${ }^{\star}$ Corresponding author: tetsuya@ @l.cis.iwate-u.ac.jp \\ Received 19 November 2012; revised 19 November 2012; accepted 27 December 2012; \\ posted 4 February 2013 (Doc. ID 174421); published 28 February 2013

\begin{abstract}
A signal-processing method is proposed in the fully interferometric three-dimensional (3D) imaging spectrometry. This processing computes a 3D interferogram, in which recorded fringe patterns do not directly reflect wavefront forms propagated from a polychromatic light source under measurement. This paper presents a procedure for signal processing including a synthesis of the 3D interferogram and retrieval of a set of spectral components of 3D images. We demonstrate retrieving 3D images for spectral components of two planar light sources by means of the proposed method. The procedure to synthesize the 3D interferogram in this method suggests the possibility of direct measurement of the 3D interferogram. (C) 2013 Optical Society of America
\end{abstract} \\ OCIS codes: $\quad 090.1995,300.6300,110.3175$.
}

\section{Introduction}

In digital holography, methods based on various phase-shifting techniques that compute complex holograms have been reported recently [1-16]. These complex holograms record wavefront information of the optical field propagated from an object under measurement. We may obtain three-dimensional (3D) spatial information of the object by the usual backpropagation techniques.

We have investigated the possibility to obtain a set of spectral components of 3D images for spatially incoherent, polychromatic objects by a fully passive interferometric technique that is strongly coupled with digital signal processing [17-23]. The principle of our method is based on the measurement of a five-dimensional (5D) spatial coherence function and

$1559-128 \mathrm{X} / 13 / 071497-08 \$ 15.00 / 0$

(C) 2013 Optical Society of America signal processing including a synthetic aperture technique. Reports of other passive interferometry include techniques to obtain a set of spectral components of two-dimensional (2D) images [24,25], various techniques to obtain $3 \mathrm{D}$ monochromatic images [26-30], and spectral tomography based on spatial coherence measurements by a rotational shear interferometer and a four-dimensional (4D) Fourier transform of the coherence function [31].

Our method can be used for a vast range of wavelengths, because special imaging devices, such as lenses, or dispersion devices, such as gratings or prisms, are not required. In addition, coherent light sources, such as a frequency tunable laser or an ultrashort pulse laser, are not necessary in our method. Since our method is based on the interferometric measurement of a partially coherent optical field propagating from a spatially incoherent, polychromatic object, it may be classified as "fully passive interferometry." 
In a previously reported method $[19,20,23]$, a $3 \mathrm{D}$ interference pattern has been computed from a dataset of originally measured interference patterns, which records a 5D spatial coherence function. This $3 \mathrm{D}$ interference pattern is proportional to a superposition of spherical waves emanating from the points on the object under measurement. Each spectrally decomposed incoherent hologram contains wavefront information of the optical field at the specific wavelength propagated from the object. By applying the technique of wavefront backpropagation to each incoherent hologram, the 3D image at a specific spectral component may be retrieved.

In this paper, we present a new method to compute an alternative $3 \mathrm{D}$ interference pattern. We call this interference pattern a hyperbolic-type volume interferogram [21]. For retrieval of the object, it is of no use the usual technique of wavefront backpropagation. Instead, a proper signal processing enables us to retrieve the object information. Then, we also show the retrieval processing of $3 \mathrm{D}$ images from the hyperbolic-type volume interferogram. In addition, the synthetic procedure of the hyperbolic-type volume interferogram indicates the possibility of getting the 3 -D interferogram directly in terms of an interferometric measurement by a fixed single detector.

In Section 2, we present a method to synthesize the hyperbolic-type volume interferogram from originally measured interference patterns. A retrieval procedure for the set of spectral components of $3 \mathrm{D}$ images from the hyperbolic-type volume interferogram is presented afterward. In Section $\underline{3}$, we demonstrate our experimental results. These results lead to the conclusion of this work, which will be stated in Section $\underline{4}$.

\section{Principle of Method}

This section presents a measurement method for the $5 \mathrm{D}$ spatial coherence function, the synthesis procedure of the hyperbolic-type volume interferogram, and retrieval of the spectral components of $3 \mathrm{D}$ images for a spatially incoherent, polychromatic object. Figure 1 shows a schematic of the twowavefront folding interferometer. This interferometer is used to measure a $5 \mathrm{D}$ spatial coherence function. The object under measurement is set on the $x-y$ stage. We take a Cartesian coordinate system

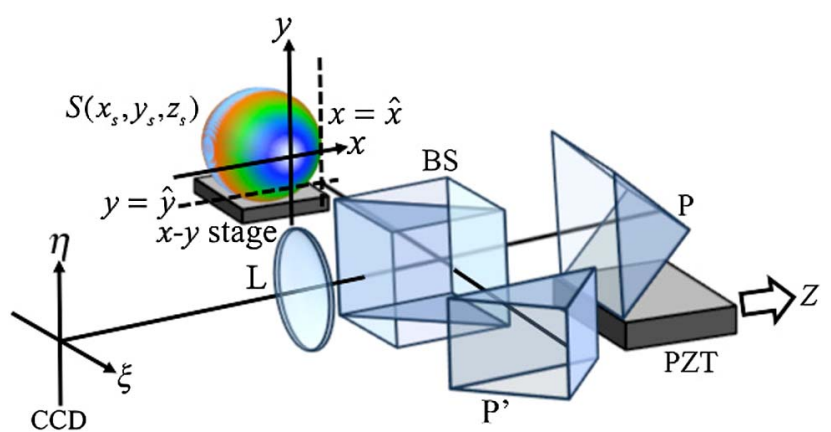

Fig. 1. (Color online) Schematic of two-wavefront folding interferometer. $(x, y, z)$ whose origin is fixed on this $x-y$ stage. The propagating optical wave from the object is split by a beam splitter (BS). Each split wavefront is reflected by right-angle prisms. Because two apexes of the prisms are set orthogonally each other, each wavefront is reversed vertically or horizontally by the prism $P$ or $P^{\prime}$. These wavefronts generate the interference pattern by superposing on the BS. The interference pattern is detected by a $2 \mathrm{D}$ array detector (CCD). We set a lens (L) between the BS and the CCD so that the $\mathrm{L}$ projects the surface of the CCD onto the apex of the prism $P^{\prime}$. We call this projected plane a virtual observation plane. The prism $P$ is mounted on the piezoelectric translator (PZT) to introduce the optical path difference $Z$ along the optical axis. The $x$ or $y$ offset of the apex of $P^{\prime}$ or $P$ is denoted as $\hat{x}$ or $\hat{y}$. During the interferometric measurement, the $x-y$ stage and PZT are moved stepwise and the CCD records the interference patterns sequentially. The whole dataset forms a 5D interferogram, and the three-dimensionally arranged $2 \mathrm{D}$ interference patterns compose it. The respective $2 \mathrm{D}$ interference pattern is called the elementally interference pattern.

We now investigate the expression of the 5D interferogram in the Cartesian coordinate system. The spatial coherence function $\Gamma\left(\boldsymbol{r}^{\prime}, \boldsymbol{r}\right)$ for the complex optical field $V(\boldsymbol{r}, t)$ at two 3D positions $\boldsymbol{r}$ and $\boldsymbol{r}^{\prime}$ at the equal time $t$ is defined as

$$
\Gamma\left(\boldsymbol{r}^{\prime}, \boldsymbol{r}\right)=\Gamma\left(\boldsymbol{r}^{\prime}, t ; \boldsymbol{r}, t\right)=\left\langle V^{*}\left(\boldsymbol{r}^{\prime}, t\right) V(\boldsymbol{r}, t)\right\rangle,
$$

where the asterisk denotes the complex conjugate and the angle bracket stands for the ensemble average. We suppress $t$ in $\Gamma$ since the optical field is stationary in time. The optical intensities at the points $\boldsymbol{r}$ and $\boldsymbol{r}^{\prime}$ are written as $\Gamma(\boldsymbol{r}, \boldsymbol{r})=\left\langle|V(\boldsymbol{r}, t)|^{2}\right\rangle$ and $\Gamma\left(\boldsymbol{r}^{\prime}, \boldsymbol{r}^{\prime}\right)=\left\langle\left|V\left(\boldsymbol{r}^{\prime}, t\right)\right|^{2}\right\rangle$. The measured 5D interferogram, which is denoted $I$, is expressed as

$$
\begin{aligned}
I(x, y, \hat{x}, \hat{y}, Z)= & \frac{1}{4}\left[\Gamma\left(\boldsymbol{r}^{\prime}, \boldsymbol{r}^{\prime}\right)+\Gamma(\boldsymbol{r}, \boldsymbol{r})+\Gamma\left(\boldsymbol{r}^{\prime}, \boldsymbol{r}\right)\right. \\
& \left.+\Gamma^{*}\left(\boldsymbol{r}^{\prime}, \boldsymbol{r}\right)\right],
\end{aligned}
$$

where the position vectors $r$ and $\boldsymbol{r}^{\prime}$ are the superposed points of the optical fields, which are reversed by prisms $P$ and $P^{\prime}$. They are written as

$$
\begin{aligned}
\boldsymbol{r} & =\left(x, 2 \hat{y}-y, z_{0}+Z\right), \\
\boldsymbol{r}^{\prime} & =\left(2 \hat{x}-x, y, z_{0}\right)
\end{aligned}
$$

where $z_{0}$ is the optical length between the virtual observation plane and the origin of the Cartesian coordinate system. We note that the $(x, y)$ origin of the Cartesian coordinate system and the origin of the coordinate system $(\xi, \eta)$ taken over the observation plane do not always coincide because of the motion of the $x-y$ stage. The relationship between $(x, y)$ coordinates and $(\xi, \eta)$ coordinates is expressed as 


$$
\begin{aligned}
& x=\xi+\hat{x}, \\
& y=\eta+\hat{y} .
\end{aligned}
$$

Upon substituting Eqs. (4) into Eqs. (3), we may express the $5 \mathrm{D}$ interferogram in Eq. $(\underline{2})$ in terms of $(\xi, \eta)$ coordinates as in the form

$I(\xi, \eta, \hat{x}, \hat{y}, Z)=\frac{1}{4}\left[\Gamma\left(\boldsymbol{r}^{\prime}, \boldsymbol{r}^{\prime}\right)+\Gamma(\boldsymbol{r}, \boldsymbol{r})+\Gamma\left(\boldsymbol{r}^{\prime}, \boldsymbol{r}\right)+\Gamma^{*}\left(\boldsymbol{r}^{\prime}, \boldsymbol{r}\right)\right]$,

where we write

$$
\begin{aligned}
r & =\left(\xi+\hat{x},-\eta+\hat{y}, z_{0}+Z\right), \\
r^{\prime} & =\left(-\xi+\hat{x}, \eta+\hat{y}, z_{0}\right) .
\end{aligned}
$$

Figure 2 shows an illustration of the 5D interferogram. In this manner, 2D elementally interference patterns are arranged three-dimensionally in accordance with the parameters $\hat{x}, \hat{y}$, and $Z$.

To retrieve the source information, we first apply a synthetic aperture technique to the $5 \mathrm{D}$ interferogram. This process computes a volume (3D) interferogram from the 5D interferogram [21]. The first step for computing this interferogram is to choose one pixel value from each elementally interference pattern according to the following selection rule:

$$
\begin{gathered}
\xi=-\hat{x}, \\
\eta=-\hat{y} .
\end{gathered}
$$

Next, we introduce new parameters,

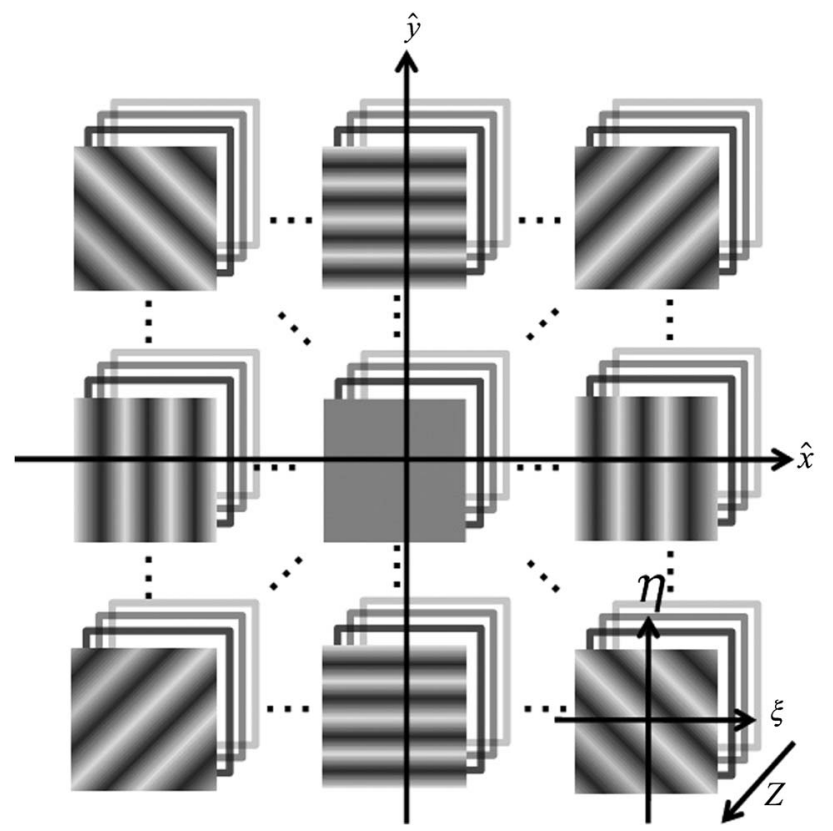

Fig. 2. Illustration of the 5D interferogram.

$$
\begin{aligned}
& X=2 \hat{x}, \\
& Y=2 \hat{y},
\end{aligned}
$$

to rearrange the selected value. The rearranged dataset forms the volume interferogram.

Our synthetic procedure consists of sampling and rearrangement, as observed above. However, this procedure is similar to a synthetic aperture processing written in [25]. Therefore, we also may call our procedure a synthetic aperture when the size of sampled pixels is as large as the size of the pinhole aperture.

According to Eqs. (6), the spatial coherence function involved in this volume interferogram is expressed as $\Gamma\left(X, 0, z_{0} ; 0, Y, z_{0}+Z\right)$. However, Fig. $\underline{3}$ shows an example of such a volume interferogram. This interferogram is calculated under the assumption that the object under measurement is a monochromatic point source of particular wavelength, located at the origin of the Cartesian coordinate system. As shown in Fig. 3, the fringe patterns across the $X-Y$ surface of this volume interferogram form a hyperbolic. In addition, we find that the orientations of the curves of the fringe patterns across the $Z-Y$ surface and the $Z-X$ surface are opposite. We call the volume interferogram that is computed with the selection rule in Eqs. (7) the hyperbolic-type volume interferogram. The selection rules and rearrangement conditions for computing the sphericaltype volume interferogram, in which the spatial coherence function involved is expressed as $\Gamma\left(0,0, z_{0}\right.$; $\left.X, Y, z_{0}+Z\right)$, are $(\xi, \eta)=(\hat{x},-\hat{y})$ and $(X, Y)=(2 \hat{x}, 2 \hat{y})$ $[20,23]$. The method to compute the hyperbolic-type volume interferogram differs from the conventional method only in the selection rules.

Because the optical field is stationary in time, the recorded spatial coherence function $\Gamma\left(X, 0, z_{0}\right.$; $\left.0, Y, z_{0}+Z\right)$ may be expressed in terms of the cross-spectral density $W_{\omega}\left(X, 0, z_{0} ; 0, Y, z_{0}+Z\right)$ as

$$
\begin{aligned}
& \Gamma\left(X, 0, z_{0} ; 0, Y, z_{0}+Z\right) \\
& \quad=\int_{0}^{\infty} W_{\omega}\left(X, 0, z_{0} ; 0, Y, z_{0}+Z\right) \mathrm{d} \omega,
\end{aligned}
$$

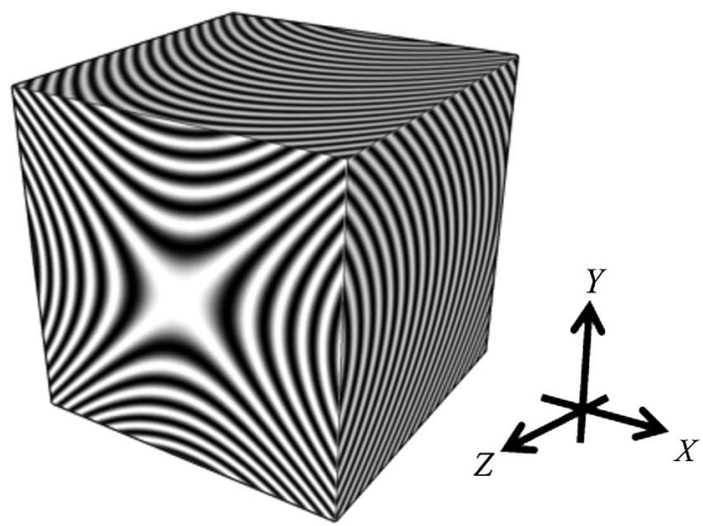

Fig. 3. Example of the hyperbolic-type volume interferogram. 
where $\omega=c k$ is the angular frequency, $c$ is speed of light in free space, and $k$ is the wave number. It is shown in Appendix A that, within the paraxial regime, the 3D cross-spectral density appearing in the right-hand-side of Eq. (9) is related to the cross-spectral density $W_{\omega}^{\left(z_{0}\right)}(X, 0 ; 0, Y) \equiv W_{\omega}\left(X, 0, z_{0}\right.$; $\left.0, Y, z_{0}\right)$ across the observation plane $z=z_{0}$ as

$$
W_{\omega}\left(X, 0, z_{0} ; 0, Y, z_{0}+Z\right)=W_{\omega}^{\left(z_{0}\right)}(X, 0 ; 0, Y) \exp (i k Z),
$$

where

$$
\begin{aligned}
W_{\omega}^{\left(z_{0}\right)}(X, 0 ; 0, Y)= & \int S_{\omega}\left(\boldsymbol{r}_{s}\right) \exp \left(i k \frac{X x_{s}-Y y_{s}}{z}\right) \\
& \times \exp \left(i k \frac{-X^{2}+Y^{2}}{2 z}\right) \mathrm{d}^{3} r_{s} .
\end{aligned}
$$

In Eq. (11), $S_{\omega}\left(\boldsymbol{r}_{s}\right)$ is the spectral density for 3D light source distribution at $\boldsymbol{r}_{s}=\left(x_{s}, y_{s}, z_{s}\right), d^{3} r_{s}=$ $d x_{s} d y_{s} d z_{s}$ and $z=z_{0}-z_{s}$. Upon substituting Eq. (10) into Eq. (9), we obtain the following relationship between the measured spatial coherence function and the cross-spectral density over the observation plane:

$$
\begin{aligned}
& \Gamma\left(X, 0, z_{0} ; 0, Y, z_{0}+Z\right) \\
& \quad=c \int_{0}^{\infty} W_{\omega}^{\left(z_{0}\right)}(X, 0 ; 0, Y) \exp (i k Z) \mathrm{d} k .
\end{aligned}
$$

It is clear that Eq. (12) may be inverted to express the cross-spectral density as the Fourier transform of the $3 \mathrm{D}$ spatial coherence function,

$$
\begin{aligned}
W_{\omega}^{\left(z_{0}\right)}(X, 0 ; 0, Y)= & \frac{1}{2 \pi c} \int \Gamma\left(X, 0, z_{0} ; 0, Y, z_{0}\right. \\
& +Z) \exp (-i k Z) \mathrm{d} Z,
\end{aligned}
$$

where the integrand is taken over the actual extension of the interferogram with respect to $Z$.

Equation (11) indicates that the spectral and 3D spatial information of the light source is recorded in the hyperbolic-type volume interferogram. We may rewrite this equation in the form

$$
\begin{aligned}
W_{\omega}^{\left(z_{0}\right)}(X, 0 ; 0, Y)= & \int S_{\omega}\left(\boldsymbol{r}_{s}\right) \exp \left[i\left(-k_{x} X+k_{y} Y\right)\right] \\
& \times \exp \left(i k \frac{-X^{2}+Y^{2}}{2 z}\right) \mathrm{d}^{3} r_{s},
\end{aligned}
$$

where $k_{x}=-k x_{s} / z$ and $k_{y}=-k y_{s} / z$ are the $x$ and $y$ components of the wave number vector $k$ of the plane wave propagating from the source direction to the center of the observation plane. We take the product of the cross-spectral density and the following function $Y_{\omega}$, which includes the retrieval depth $z^{\prime}$ and wave number $k=\omega / c$ :

$$
Y_{\omega}\left(X, Y, z^{\prime}\right)=\exp \left(-i k \frac{-X^{2}+Y^{2}}{2 z^{\prime}}\right)
$$

The monochromatic component of the retrieved image $O_{\omega}\left(x, y, z^{\prime}\right)$ is expressed as

$$
\begin{aligned}
O_{\omega}\left(x, y, z^{\prime}\right)= & \int W_{\omega}^{\left(z_{0}\right)}(X, 0 ; 0, Y) Y_{\omega}\left(X, Y, z^{\prime}\right) \\
& \times \exp \left[-i\left(-k_{x}^{\prime} X+k_{y}^{\prime} Y\right)\right] \mathrm{d}^{3} r_{s},
\end{aligned}
$$

where $k^{\prime}=-k x / z^{\prime}$ and $k^{\prime}=-k y / z^{\prime}$. The retrieved image is focused on the $z=z^{\prime}$ plane, while objects located at other depths are defocused. This phenomenon is well known in conventional holographic 3D imaging.

The fields of view (FOVs) along the $x$ and $y$ directions, denoted $x_{\max }$ and $y_{\max }$, are expressed as $x_{\max }=$ $z^{\prime} \lambda / \Delta l_{x}$ and $y_{\max }=z^{\prime} \lambda / \Delta l_{y}$, where $\Delta l_{x}$ and $\Delta l_{y}$ are the pixel sizes of the complex hologram in the $x$ and $y$ directions. These FOVs are as large as the FOVs of the retrieval images in the conventional method [20], because the retrieval procedure from the hyperbolic-type volume interferogram differs from the conventional method only in the sign of $X^{2}$ in the quadratic phase factor and $k^{\prime}$ in the linear phase factor.

\section{Experiment}

To verify our method described in the previous section, we demonstrate the experimental results for retrieving 3D images for spectral components of two planar light sources. The shapes and continuous spectra of these sources are different. These planar light sources are located at different depths. The planar sources to be measured are denoted $S_{1}$ and $S_{2}$. They are composed of guiding lights of light-emitting diodes through acrylic rods whose front ends are shaped as triangles and squares. The surfaces of the front ends are roughened. Figure 4 shows a front view of $S_{1}$ and $S_{2}$. The optical depth of $S_{1}$, denoted as $z_{1}$, is $95 \mathrm{~mm}$, and that of $S_{2}, z_{2}$ is $82 \mathrm{~mm}$. Figure 5 shows spectral profiles of the light sources $S_{1}$ and $S_{2}$. Each spectral profile is measured by Fourier transform spectroscopy separately and normalized as the peak intensity becomes unity. Spectral resolutions of these profiles are $\Delta k / 2 \pi=61.09 \mathrm{~cm}^{-1}$. The typical wavelengths of the continuous spectra of those planar light sources are approximately $\lambda=$ $458 \mathrm{~nm}$ for $S_{1}$ and $643 \mathrm{~nm}$ for $S_{2}$.

We record the 5D interferogram using the twowavefront folding interferometer. The numbers of steps and intervals are 64 and $12.9 \mu \mathrm{m}$ for the $x-y$ stage and 64 and $0.08 \mu \mathrm{m}$ for the PZT. Then, the $5 \mathrm{D}$ interferogram, measured by this experiment, consists of $64 \times 64 \times 64$ elementally interference patterns. We then compute the hyperbolic-type volume interferogram based on the new selection rule in Eqs. (7). The obtained volume interferogram is shown in Fig. 6 . The quarter part of the interfeogram 


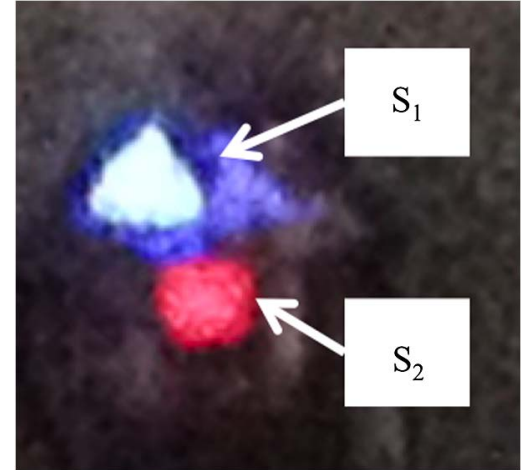

Fig. 4. (Color online) Front view of $S_{1}$ and $S_{2}$.

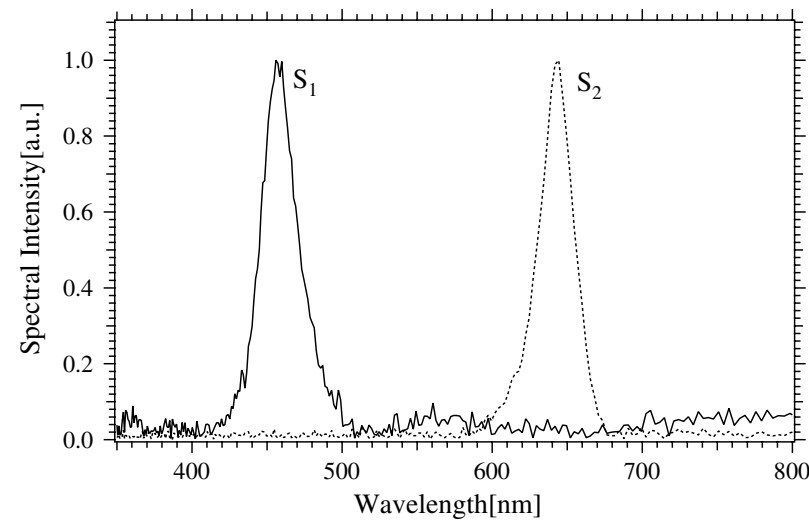

Fig. 5. Spectral profile of $S_{1}$ and $S_{2}$. These spectral profile are measured separately by Fourier transform spectrometry.

is removed to show the inner fringe arrangement. In Fig. 6 , high-contrast fringe patterns along the direction of $Z$ are located near the optical axis (center of the volume interferogram). This corresponds to the coherence area of the partially coherent optical field under observation.

The spectral profile measured across the observation plane is shown in Fig. 7. This is obtained by taking the Fourier transform of the intensity distribution at the center of the volume interferogram with respect to $Z$. The spectral resolution of this profile is $\Delta k / 2 \pi=992.06 \mathrm{~cm}^{-1}$. The spectral peaks are located near 458 and $630 \mathrm{~nm}$. Within the spectral resolution, these peaks agree with the peak wavelengths of the respective light sources, given by Fig. 5 . In addition, by taking the Fourier transform with respect to $Z$ of the volume interferogram, we obtain a set of the cross-spectral densities in Eq. (13). Figure 8 shows the cross-spectral densities at $\lambda=$ $458 \mathrm{~nm}$ and $\lambda=630 \mathrm{~nm}$. Figures $\underline{8(\mathrm{a})}$ and $8(\mathrm{~b})$ show the absolute value distribution and the phase distribution at $\lambda=458 \mathrm{~nm}$, and (c) and (d) show those at $\lambda=630 \mathrm{~nm}$. Figure 9 shows a comparison of the in-focus images obtaine $\bar{d}$ by the present method and those obtained by the conventional method proposed in [20]. Figures 9(a) and 9(b) show the retrieved images at $\lambda=458 \overline{\mathrm{nm}}$ and $\overline{z^{\prime}}=z_{1}$, and at $\lambda=630 \mathrm{~nm}$ and $z^{\prime}=z_{2}$. Figures $9(\mathrm{c})$ and $9(\mathrm{~d})$ show

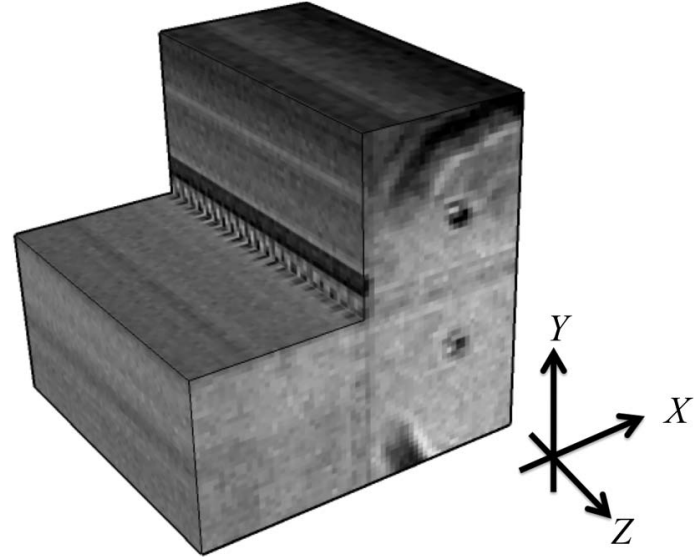

Fig. 6. Hyperbolic-type volume interferogram computed by the new selection rule in Eqs. (7). The quarter part of the interferogram is removed to show inner fringe arrangement.

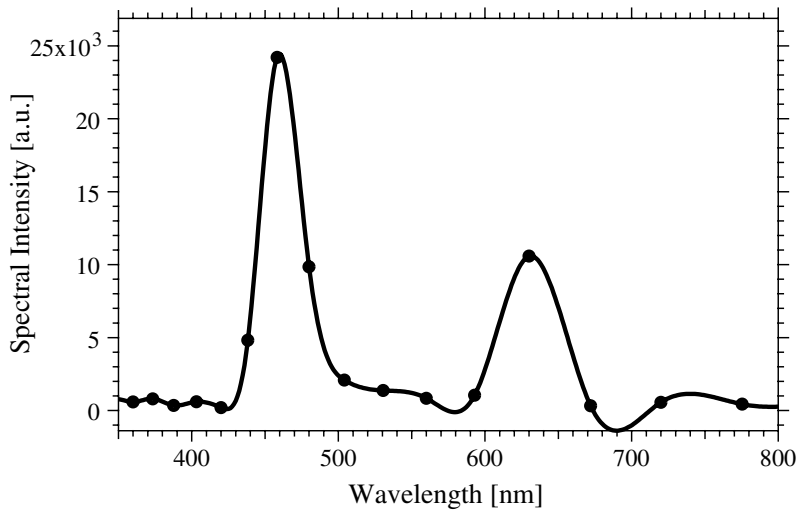

Fig. 7. Spectral profile of the optical field over the observation plane.

retrieved images by the conventional method [20] under the same conditions as in Figs. 9(a) and $\underline{9(\mathrm{~b})}$. These images are obtained from the same $5 \mathrm{D}$ interferogram. We find that the images retrieved from the hyperbolic-type volume interferogram are similar to the images retrieved from the spherical-type volume interferogram. These shapes of images agree with the shape shown in Fig. 4. Since the vertical variations of both methods are very slow, we could not confirm these depth resolutions. This is because the distance between the optical sources to be measured and the observation plane is too long for the sizes of the complex holograms. From these experimental results, we conclude that the object information for the planar light sources can be retrieved from the hyperbolic-type volume interferogram. The improvement of the depth resolution for retrieving the image of the object having horizontal width is a subject to be explored in the future.

\section{Conclusion}

We have presented the method to synthesize the hyperbolic-type volume interferogram. This technique is based on a new selection rule and data rearrangement. The fringe patterns of the hyperbolic-type 


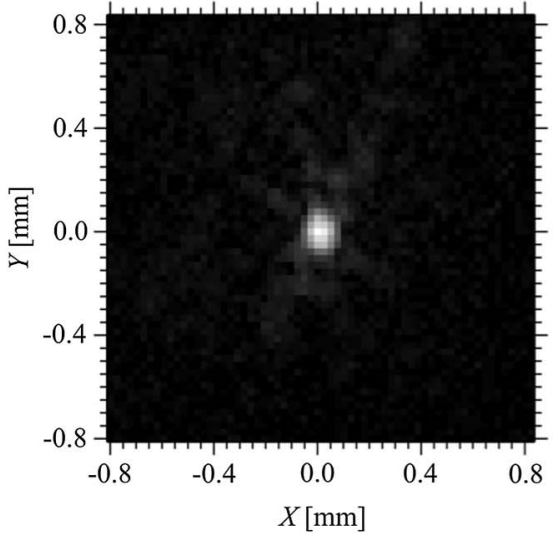

(a)

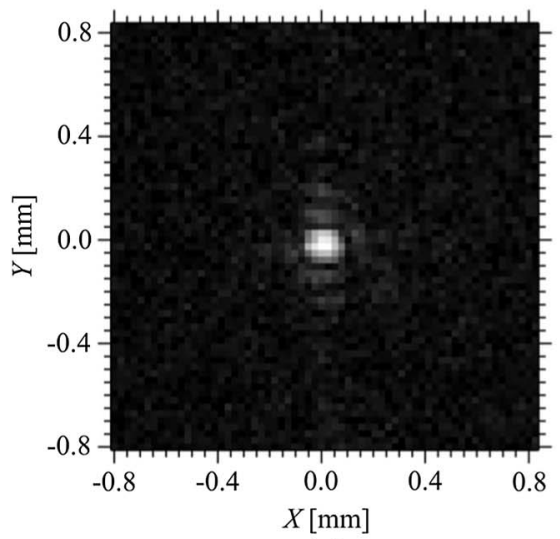

(c)

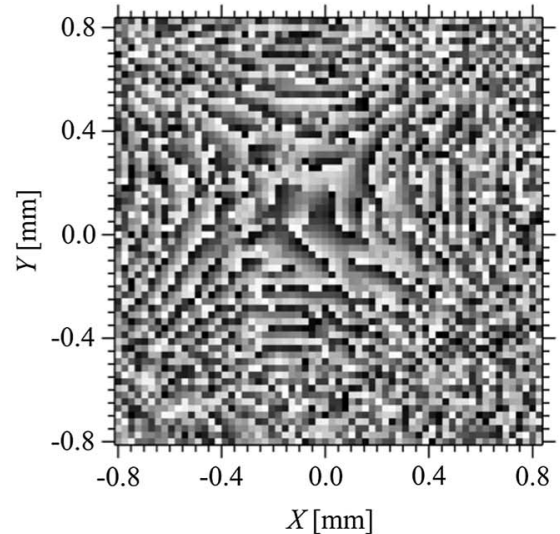

(b)

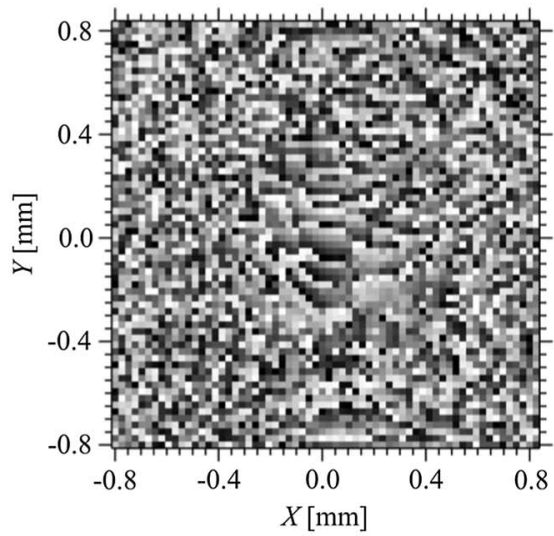

(d)

Fig. 8. Absolute value image and phase distribution of the cross spectral density (a), (b) at $\lambda=458 \mathrm{~nm}$, and (c), (d) at $\lambda=630 \mathrm{~nm}$.

volume interferogram do not directly correspond to wavefront forms propagated from the object to be measured. We also proposed the method for retrieving a set of spectral components of $3 \mathrm{D}$ images from the hyperbolic-type volume interferogram. From our experimental results, we may state that it is possible to retrieve the object information of spatially incoherent, polychromatic objects from the hyperbolic-type volume interferogram.

The selection rule to obtain the hyperbolic-type volume interferogram chooses one interference intensity at a particular point on each elementally interference pattern. In the coordinate system taken over the observation plane, the selected points to generate the hyperbolic-type volume interferogram are written as $(-\hat{x},-\hat{y})$. However, if the same points are viewed from the Cartesian coordinate system, one finds that the selected points locate $(0,0)$. Thus, these points are identical and fixed at the $(x, y)$ origin of the Cartesian coordinate system. This suggests a possibility of using a single detector instead of a $2 \mathrm{D}$ detector array for direct acquisition of the hyperbolictype volume interferogram, because the measure points always coincide with the $x-y$ origin of the Cartesian coordinate system. For direct acquisition of the spherical-type volume interferogram, the detector needs to move over the observation plane, though for direct acquisition of the hyperbolic-type volume interferogram, the detector only has to be fixed. We plan to report the validation of such an idea for an interferometer in the future.

\section{Appendix A: Derivation of Eq. (10)}

The cross-spectral density $W_{\omega}\left(\boldsymbol{r}^{\prime}, \boldsymbol{r}\right)$ for the monochromatic complex optical field $U_{\omega}(r)$ at a pair of position vectors $\boldsymbol{r}^{\prime}$ and $\boldsymbol{r}$ is written as

$$
\begin{aligned}
W_{\omega}\left(\boldsymbol{r}^{\prime}, \boldsymbol{r}\right) & =\left\langle U^{*}\left(\boldsymbol{r}^{\prime}, t\right) U(\boldsymbol{r}, t)\right\rangle \\
& =\int \frac{S_{\omega}\left(\boldsymbol{r}_{s}\right)}{r r^{\prime}} \exp \left[i k\left(r-r^{\prime}\right)\right] \mathrm{d}^{3} r_{s},
\end{aligned}
$$

where $\langle\cdots\rangle$ stands for the ensemble average, the asterisk denotes the complex conjugate, $r^{\prime}=\left|\boldsymbol{r}^{\prime}-\boldsymbol{r}_{s}\right|$, and $r=\left|\boldsymbol{r}-\boldsymbol{r}_{s}\right|$. In Eq. (A1), $\boldsymbol{S}_{\omega}\left(\boldsymbol{r}_{s}\right)$ denotes the spectral density with angular frequency $\omega$ for the spatially incoherent optical source at $\boldsymbol{r}_{s}=\left(x_{s}, y_{s}, z_{s}\right)$, $k=\omega / c$ is the wave number, and $c$ is the speed of the light in free space. In the sense of paraxial resume, we assume that $\boldsymbol{r}^{\prime}$ and $\boldsymbol{r}$ are far enough away from the object and are located near the optical axis. If we set $\boldsymbol{r}^{\prime}$ and $\boldsymbol{r}$ as

$$
r^{\prime}=\left(X, 0, z_{0}\right)
$$




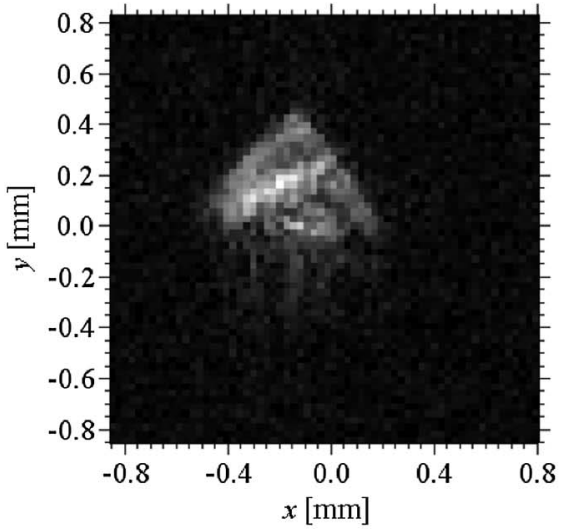

(a)

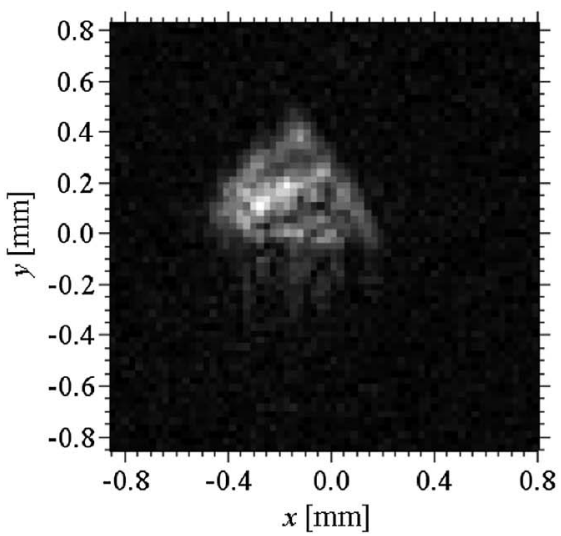

(c)

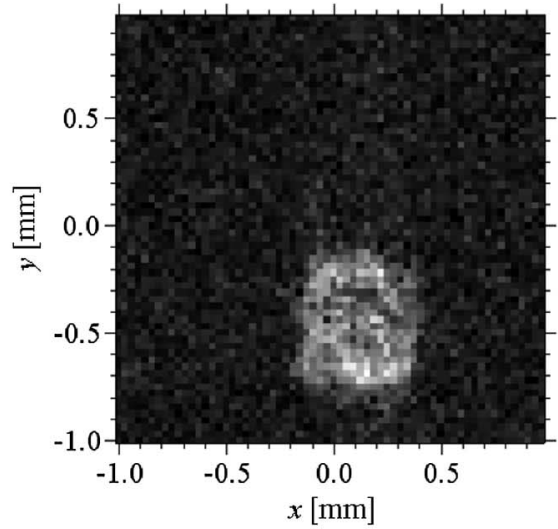

(b)

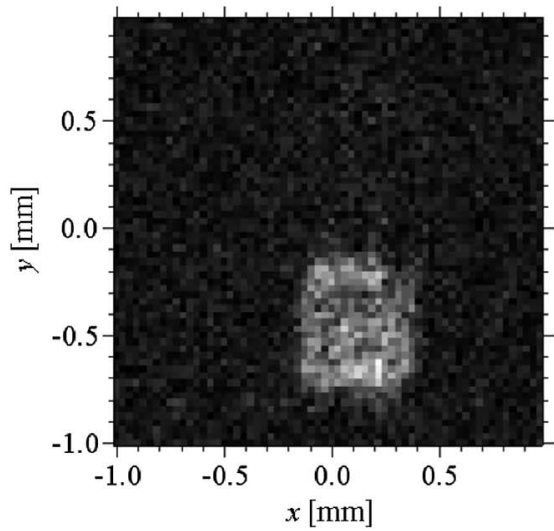

(d)

Fig. 9. Retrieved images by the proposed method at (a) $\lambda=458 \mathrm{~nm}$ and $z^{\prime}=95 \mathrm{~mm}$, (b) $\lambda=630 \mathrm{~nm}$ and $z^{\prime}=82 \mathrm{~mm}$, and those by the conventional method, at (c) $\lambda=458 \mathrm{~nm}$ and $z^{\prime}=95 \mathrm{~mm}$, and (d) $\lambda=630 \mathrm{~nm}$ and $z^{\prime}=82 \mathrm{~mm}$.

and

$$
\boldsymbol{r}=\left(0, Y, z_{0}+Z\right)
$$

then $r^{\prime}$ and $r$ in Eq. (1ㅗ $)$ are written as

$$
\begin{aligned}
& r^{\prime}=\sqrt{\left(X-x_{s}\right)^{2}+y_{s}^{2}+\left(z_{0}-z_{s}\right)^{2}}, \\
& r=\sqrt{x_{s}^{2}+\left(Y-y_{s}\right)^{2}+\left(z_{0}+Z-z_{s}\right)^{2}} .
\end{aligned}
$$

Within the paraxial approximation, Eqs. (스) are expressed as

$$
\begin{aligned}
& r^{\prime}=z_{0}-z_{s}+\frac{\left(X-x_{s}\right)^{2}+y_{s}^{2}}{2\left(z_{0}-z_{s}\right)}, \\
& r=z_{0}+Z-z_{s}+\frac{x_{s}^{2}+\left(Y-y_{s}\right)^{2}}{2\left(z_{0}+Z-z_{s}\right)} .
\end{aligned}
$$

By assuming $z_{0} \gg Z$, Eq. (A1) is rewritten as

$$
\begin{aligned}
W_{\omega}\left(\boldsymbol{r}^{\prime}, \boldsymbol{r}\right)= & \exp (i k Z) \int S_{\omega}\left(\boldsymbol{r}_{s}\right) \exp \left(i k \frac{X x_{s}-Y y_{s}}{z}\right) \\
& \times \exp \left(i k \frac{-X^{2}+Y^{2}}{2 z}\right) \mathrm{d}^{3} r_{s},
\end{aligned}
$$

where $z=z_{0}-z_{s}$. For simplicity, the distances $r$ and $r^{\prime}$ in the denominator in Eq. (A1) have been absorbed in the spectral density $S_{\omega}$. Similarly, we consider the cross-spectral density $W_{\omega}\left(\boldsymbol{r}^{\prime}, \boldsymbol{r}^{\prime \prime}\right)$, where the point $\boldsymbol{r}^{\prime \prime}$ is the position of $\boldsymbol{r}$ shifting to $Z=0$ is written as

$$
\begin{aligned}
W_{\omega}\left(\boldsymbol{r}^{\prime}, \boldsymbol{r}^{\prime \prime}\right)= & \int S_{\omega}\left(\boldsymbol{r}_{s}\right) \exp \left(i k \frac{X x_{s}-Y y_{s}}{z}\right) \\
& \times \exp \left(i k \frac{-X^{2}+Y^{2}}{2 z}\right) \mathrm{d}^{3} r_{s} .
\end{aligned}
$$

By comparing Eq. (A5) with (A6), one obtains the following equation in Eq. (10):

$$
W_{\omega}\left(\boldsymbol{r}^{\prime}, \boldsymbol{r}\right)=W_{\omega}\left(\boldsymbol{r}^{\prime}, \boldsymbol{r}^{\prime \prime}\right) \exp (i k Z) .
$$


This work is supported in part by the Research Foundation for Opt-Science and Technology, and by Grants-in-Aid for Scientific Research from the Japan Society for the Promotion of Science (22560031).

\section{References}

1. I. Yamaguchi and T. Zhang, "Phase-shifting digital holography," Opt. Lett. 22, 1268-1270 (1997).

2. Y. Zhang, G. Pedrini, W. Osten, and H. J. Tiziani, "Reconstruction of in-line digital holograms from two intensity measurements," Opt. Lett. 29, 1787-1789 (2004).

3. Y. Awatsuji, A. Fujii, T. Kubota, and O. Matoba, "Parallel three-step phase shifting digital holography," Appl. Opt. 45, 2995-3002 (2006).

4. R. Tumbar, D. L. Marks, and D. J. Brady, "Robust, common path, phase shifting interferometer and optical profilometer," Appl. Opt. 47, B32-B43 (2008).

5. G. Situ, J. P. Ryle, U. Gopinathan, and J. T. Sheridan, "Generalized in-line digital holographic technique based on intensity measurements at two different planes," Appl. Opt. 47, 711-717 (2008)

6. J. Hahn, H. Kim, S.-W. Cho, and B. Lee, "Phase-shifting interferometry with genetic algorithm-based twin image noise elimination," Appl. Opt. 47, 4068-4076 (2008).

7. Y. Awatsuji, T. Tahara, A. Kaneno, T. Koyama, K. Nishio, S. Ura, T. Kubota, and O. Matoba, "Parallel two-step phaseshifting digital holography," Appl. Opt. 47, D183-D189 (2008).

8. M.-L. Cruz, A. Castro, and V. Arrizón, "Phase shifting digital holography implemented with a twisted-nematic liquidcrystal display," Appl. Opt. 48, 6907-6912 (2009).

9. T. Nomura and M. Imbe, "Single-exposure phase-shifting digital holography using a random-phase reference wave," Opt. Lett. 35, 2281-2283 (2010).

10. T. Tahara, Y. Awatsuji, K. Noshio, S. Ura, T. Kubota, and O. Matoba, "Comparative analysis and quantitative evaluation of the field of view and the viewing zone of single-shot phase-shifting digital holography using space-division multiplexing," Opt. Rev. 17, 519-524 (2010).

11. T. Kiire, S. Nakadate, M. Shibuya, and T. Yatagai, "Quadrature phase-shifting interferometer using spatial carrier," Opt. Rev. 18, 103-106 (2011).

12. T. Kakue, R. Yonesaka, T. Tahara, Y. Awatsuji, K. Nishio, S. Ura, T. Kubota, and O. Matoba, "High-speed phase imaging by parallel phase-shifting digital holography," Opt. Lett. 36, 4131-4133 (2011).

13. T. Kiire, S. Nakadate, M. Shibuya, and T. Yatagai, "Threedimensional displacement measurement for diffuse object using phase-shifting digital holography with polarization imaging camera," Appl. Opt. 50, H189-H194 (2011).

14. T. Tahara, Y. Shimozato, Y. Awatsuji, K. Nishio, S. Ura, O. Matoba, and T. Kubota, "Spatial-carrier phase-shifting digital holography utilizing spatial frequency analysis for the correction of the phase-shift error," Opt. Lett. 37, 148-150 (2012).

15. M. Lin, K. Nitta, O. Matoba, and Y. Awatsuji, "Parallel phase-shifting digital holography with adaptive function using phase-mode spatial light modulator," Appl. Opt. 51, 2633-2637 (2012).

16. P. Földesy, "Terahertz single-shot quadrate phase-shifting interferometry," Opt. Lett. 37, 4044-4046 (2012).

17. K. Yoshimori, "Interferometric spectral imaging for threedimensional objects illuminated by a natural light source," J. Opt. Soc. Am. A 18, 765-770 (2001).

18. K. Yoshimori, "Passive digital multispectral holography based on synthesis of coherence function," Proc. SPIE 6252, 625221 (2006).

19. M. Sasamoto and K. Yoshimori, "First experimental report on fully passive interferometric three-dimensional imaging spectrometry," Jpn. J. Appl. Phys. 48, 09LB03 (2009).

20. M. Sasamoto and K. Yoshimori, "Three-dimensional imaging spectrometry by fully passive interferometry," Opt. Rev. 19, 29-33 (2012).

21. T. Hashimoto and K. Yoshimori, "Fully interferometric threedimensional imaging spectrometry using hyperbolic-type volume interferogram," in Digital Holography and ThreeDimensional Imaging, OSA Techinal Digest (CD) (Optical Society of America, 2011), paper DWC38.

22. K. Yoshimori, "Digital holographic three-dimensional imaging spectrometry," in Digital Holography and Three-Dimensional Imaging, OSA Technical Digest (Optical Society of America, 2012), paper DW1C.1.

23. S. Teeranutranont and K. Yoshimori, "Digital holographic three-dimensional imaging spectrometry," Appl. Opt. 52, A388-A396 (2013)

24. K. Itoh and Y. Ohtsuka, "Fourier-transform spectral imaging: retrieval of source information from three-dimensional spatial coherence," J. Opt. Soc. Am. A 3, 94-100 (1986).

25. K. Itoh, T. Inoue, T. Yoshida, and Y. Ichioka, "Interferometric supermultispectral imaging," Appl. Opt. 29, 1625-1630 (1990).

26. J. Rosen and A. Yariv, "General theorem of spatial coherence: application to three-dimensional imaging," J. Opt. Soc. Am. A 13, 2091-2095 (1996).

27. D. L. Marks, R. A. Stack, and D. J. Brady, "Three-dimensional coherence imaging in the Fresnel domain," Appl. Opt. 38, 1332-1342 (1999).

28. H. Arimoto, K. Yoshimori, and K. Itoh, "Retrieval of the cross-spectral density propagating in free space," J. Opt. Soc. Am. A 16, 2447-2452 (1999).

29. H. Arimoto, K. Yoshimori, and K. Itoh, "Interferometric three-dimensional imaging based on retrieval of generalized radiance distribution,” Opt. Rev. 7, 25-33 (2000).

30. J. Rosen and G. Brooker, "Digital spatially incoherent Fresnel holography," Opt. Lett. 32, 912-914 (2007).

31. D. L. Marks, M. Fetterman, R. A. Stack, and D. J. Brady, "Spectral tomography from spatial coherence measurements," Proc. SPIE 3920, 48-55 (2000). 